\title{
UAV-based antenna measurement and diagnostics for circularly polarized antenna arrays
}

\author{
M. Garcia-Fernandez, Y. Alvarez-Lopez, F. Las-Heras \\ Area of Signal Theory and Communications, University of Oviedo, Gijon (Spain). Email: mariagarcia@tsc.uniovi.es
}

\begin{abstract}
The main goal of this contribution is to present the application of an Unmanned Aerial System for Antenna Measurement (UASAM) for analyzing circularly polarized antenna arrays. UASAM can be used for in-situ antenna measurement and diagnostics. It makes use of a power detector onboard an Unmanned Aerial Vehicle (UAV) with a cm-level accuracy positioning system, which allows to acquire measurements in the near field region. Thus, it overcomes practical drawbacks of other UAV-based antenna measurement systems that work in the far field region. Its performance for providing antenna diagnostics of an array composed by two circularly polarized antennas with reverse handedness is analyzed, comparing it with anechoic chamber results.
\end{abstract}

\section{INTRODUCTION}

Recent advances in the development of Unmanned Aerial Vehicles (UAVs) have led to new applications in several fields such as electromagnetic radiation measurements [1],[2]. UAVbased antenna measurement solutions allow to take measurements at the antenna operational location. Therefore, they take into account the influence of the surroundings at the expense of being less accurate than anechoic chamber measurements. They are also less expensive and easier to use than similar solutions based on manned aircrafts.

These systems can be classified according to: i) the nature of the RF devices onboard the UAV (a power detector, a spectrum analyzer or even a transmitter); and ii) the region of operation (Near-Field, NF, or Far-Field, FF). Most stateof-the-art systems acquire the antenna radiation pattern in the FF region, which might start far away from the Antenna Under Test (AUT) for low frequencies. To overcome their main drawbacks, regarding flight restrictions and autonomy issues, we have recently proposed an Unmanned Aerial System for Antenna Measurement (UASAM) that works in the NF region and uses a low cost power detector [3],[4]. UASAM autonomously follows a predefined measurement grid, sending the geo-referred measurements to a ground control station, where they are post-processed to obtain the AUT aperture fields (providing antenna diagnostics information) and the radiation pattern (through a NF-FF transformation).

UASAM has been successfully employed for measuring linearly polarized antennas and arrays up to $C$ band. The main goal of this contribution is to briefly describe UASAM main features as well as the last results of antenna diagnostics for circularly polarized arrays. Furthermore, these results are compared with those based on anechoic chamber measurements.

\section{UASAM ARCHITECTURE}

UASAM is composed by a UAV, a ground control station and a communication system between them. The UAV includes: i) a flight controller, ii) common positioning sensors (Inertial Measurement Units, barometer and Global Navigation Satellite Systems -GNSS-), iii) an accurate positioning subsystem (based on a Real Time Kinematic -RTK- beacon and a laser rangefinder) to provide cm-level accuracy positioning, and iv) the antenna measurement subsystem (a power detector and a probe antenna). The RTK system makes also use of an on-ground RTK beacon, which provides GNSS corrections. The communication system frequencies are selected so as not to interfere with the measurements (in this case, $433 \mathrm{MHz}$ and $2.4 \mathrm{GHz}$ ). A picture of the UASAM prototype taking the measurements of this contribution is shown in Fig. 1 .

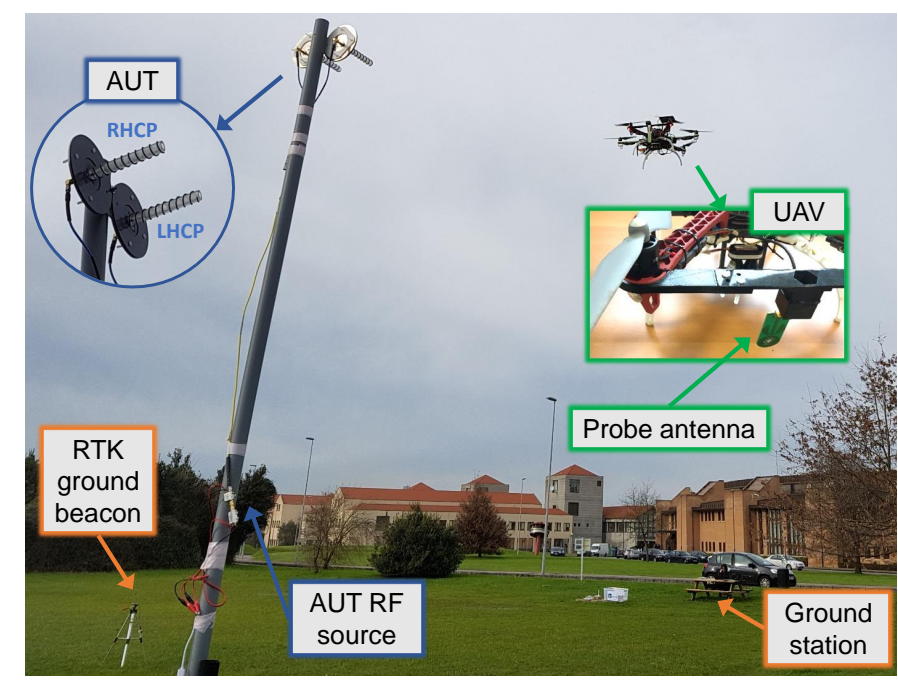

Fig. 1. UASAM prototype for measuring an array of two circularly polarized antennas with reverse handedness (right hand and left hand circular polarization -RHCP and LHCP-).

Due to the use of a power detector, phase cannot be measured. Thus, a phase retrieval technique called phaseless Sources Reconstruction Method (pSRM) is used. This technique allows to recover the AUT aperture fields as long as the measurements are acquired in at least two measurement grids (e.g. two parallel planar surfaces). Furthermore, in order to be able to measure antennas working at frequencies higher than $1 \mathrm{GHz}, \mathrm{cm}$-level accuracy positioning is required. 


\section{EXPERIMENTAL VALIDATION}

\section{A. Setup description}

The AUT is an array composed by two circularly polarized antennas with reverse handedness placed on top of a $3-\mathrm{m}$ height mast. This array is fed with an oscillator working at $4.65 \mathrm{GHz}$. The probe antenna is a monopole that has an omnidireccional pattern in the H-plane. Due to its linear polarization, it must be placed at two orthogonal directions to be able to measure both the vertical and horizontal electric field components ( $E_{y}$ and $E_{x}$ in this work).

The measurement grid is composed by two parallel planar surfaces of $6 \mathrm{~m}$ width at 3.2 and $4.5 \mathrm{~m}$ away from the AUT, with the height ranging from 2 to $4.5 \mathrm{~m}$ in $7 \mathrm{~cm}$ steps.

\section{B. Near field measurements comparison}

In order to test the performance of UASAM, the AUT has also been measured in an anechoic chamber, obtaining an electromagnetic (EM) model of the AUT based on equivalent currents. With this model and the flight path coordinates, the NF amplitude has been simulated at the UAV positions.

$E_{y}$ and $E_{x} \mathrm{NF}$ components are compared in Fig. 2 for the measurement grid placed at $3.2 \mathrm{~m}$ distance. There is a good agreement between simulated and measured NF, both showing three and two lobes in the case of $E_{y}$ and $E_{x}$, respectively.

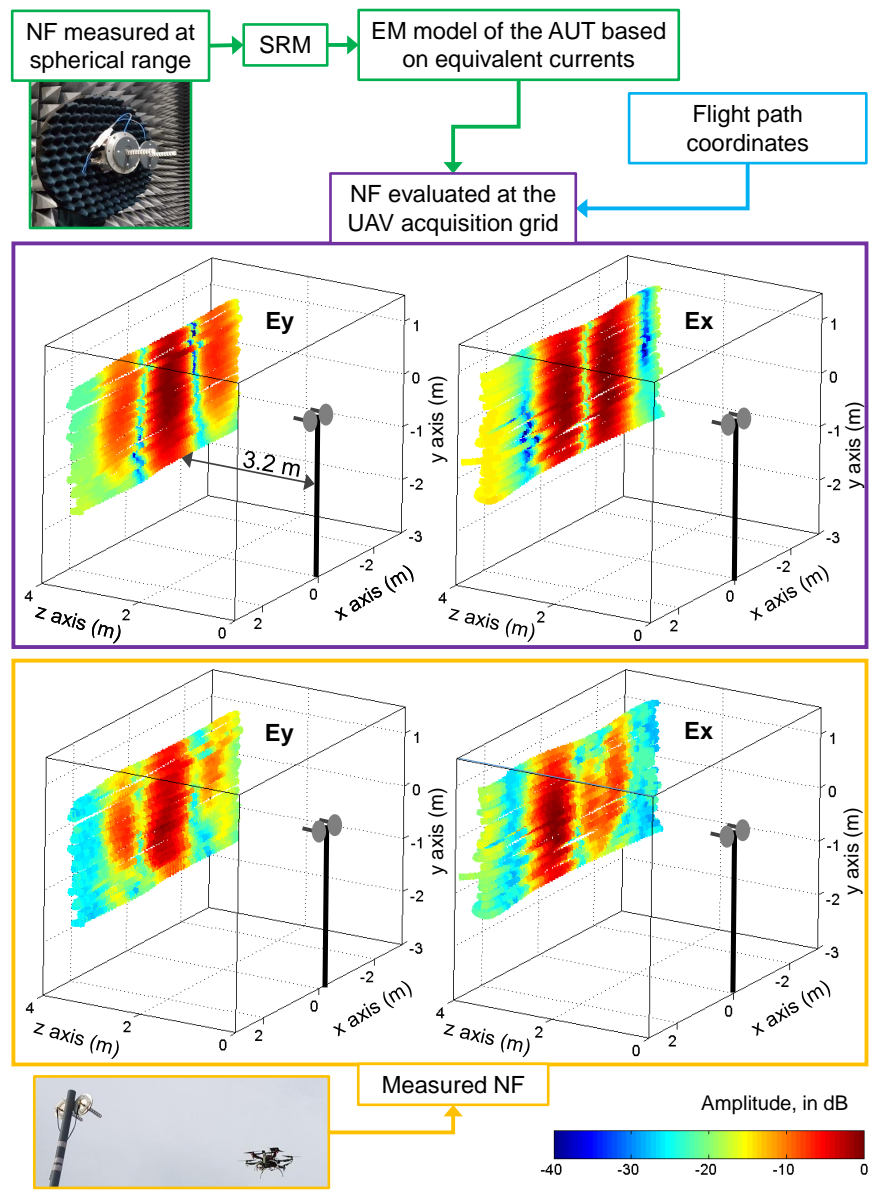

Fig. 2. Simulated and measured NF amplitude.

\section{Antenna diagnostics comparison}

From NF amplitude measurements, an equivalent magnetic currents distribution on the AUT aperture plane is recovered using pSRM. Recovered $M_{y}$ (from $E_{x}$ ) and $M_{x}$ (from $E_{y}$ ) are then expressed as circular polarization components, $M_{R H C}$ and $M_{L H C}$.

Fig. 3 shows the reconstructed equivalent currents. It can be seen that the circular polarization associated to each antenna is properly retrieved.
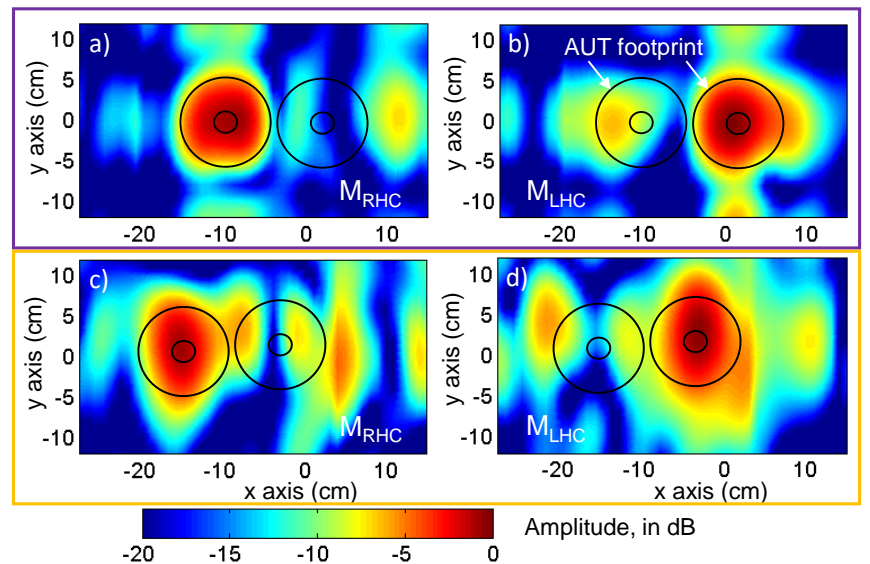

Fig. 3. Equivalent magnetic currents (circular components) distribution at the AUT aperture: from simulated NF (a, b) and from measured NF (c, d).

\section{CONCLUSION}

In this contribution, the application of a new system to perform in-situ NF antenna measurements and diagnostics (UASAM) for measuring circularly polarized arrays has been presented. UASAM can be used for several applications such as rapid assessment of mobile networks base stations coverage, location of malfunctioning elements in arrays, among others.

\section{ACKNOWLEDGEMENT}

This work has been partially supported by Spanish government under project TEC2014-55290-JIN and grant FPU15/06341.

\section{REFERENCES}

[1] F. Paonessa et al., "UAV-based pattern measurement of the SKALA", in Proc. IEEE Int. Symp. Antennas Prop. \& USNC/URSI Nat. Radio Sci. Meet. (APSURSI), pp. 1372-1373, July 2015.

[2] E. Teng, J. D. Falcao, C. R. Dominguez, F. Mokaya, P. Zhang, B. Iannucci, "Aerial sensing and characterization of three-dimensional RF fields", in Proc. 2nd Int. Workshop Robot. Sensor Netw., pp. 1-6, April 2015.

[3] M. Garcia-Fernandez, Y. Alvarez-Lopez, A. Arboleya, B. GonzalezValdes, Y. Rodriguez-Vaqueiro, M. E. de Cos, F. Las Heras, "Antenna Diagnostics and Characterization using Unmanned Aerial Vehicles", IEEE Access, vol. 5, pp. 23563-23575, September 2017.

[4] Y. Alvarez-Lopez, M. Garcia-Fernandez, F. Las Heras, A. ArboleyaArboleya, B. Gonzalez-Valdes, Y. Rodriguez-Vaqueiro, A. Garcia-Pino, "Airborne system and method for the characterization and measurement of antennas or radiating systems", Spanish Patent App. P201700209, 2017. 